\title{
Does an increase in serum FGF21 level predict 28-day mortality of critical patients with sepsis and ARDS?
}

\author{
Xing Li ${ }^{\dagger}$, Hua Shen ${ }^{\dagger}$, Tinghong Zhou, Xiaoyu Cao, Ying Chen, Yan Liang, Ting Lu, Jiafen He, Zhoulin Dou, \\ Chuankai Liu, Yong Tang ${ }^{*}$ and Zexiang Zhu* (D)
}

\begin{abstract}
Background: Sepsis may be accompanied by acute respiratory distress syndrome (ARDS) in patients admitted to intensive care units (ICUs). It is essential to identify prognostic biomarkers in patients with sepsis and ARDS.

Objective: Determine whether changes in the level of serum fibroblast growth factor 21 (FGF21) can predict the 28-day mortality of ICU patients with sepsis and ARDS.

Methods: Consecutive sepsis patients were divided into two groups (Sepsis + ARDS and Sepsis-only), and the Sepsis + ARDS group was further classified as survivors or non-survivors. Demographic data and comorbidities were recorded. The Sequential Organ Failure Assessment (SOFA) score and serum levels of cytokines and other biomarkers were recorded 3 times after admission. Multiple Cox proportional hazards regression was used to identify risk factors associated with 28-day mortality in the Sepsis + ARDS group. Multivariate receiver operating characteristic curve analysis was used to assess the different predictive value of FGF21 and SOFA.

Results: The Sepsis + ARDS group had a greater baseline SOFA score and serum levels of cytokines and other biomarkers than the Sepsis-only group; the serum level of FGF21 was almost twofold greater in the Sepsis + ARDS group $(P<0.05)$. Non-survivors in the Sepsis + ARDS group had an almost fourfold greater level of FGF21 than survivors in this group $(P<0.05)$. The serum level of FGF21 persistently increased from the baseline to the peak of shock and death in the non-survivors, but persistently decreased in survivors $(P<0.05)$. Changes in the serum FGF21 level between different time points were independent risk factors for mortality. No statistical difference was observed between the AUC of FGF21 and SOFA at baseline.
\end{abstract}

Conclusion: A large increase of serum FGF21 level from baseline is associated with 28-day mortality in ICU patients with sepsis and ARDS.

\section{Background}

Sepsis is defined as a life-threatening organ dysfunction caused by the host's deregulated response to an infection

\footnotetext{
*Correspondence: 407891521@qq.com; Zhuzexiang12@163.com †Xing Li and Hua Shen have contributed equally to this work and should be regarded as co-first authors

Department of Critical Care, Changsha of Traditional Chinese Medicine Hospital, No. 22, Xingsha Road, Changsha 410010, Hunan Province, People's Republic of China
}

[1]. Sepsis may lead to acute respiratory distress syndrome (ARDS), a condition characterized by acute, diffuse, and inflammatory lung injury that leads to increased non-hydrostatic extravascular lung water, reduced lung compliance, and severe hypoxemia [2].

Despite therapeutic innovations, the mortality rate of patients with sepsis is as high as 25-30\% [3] and the mortality rate of patients with ARDS can reach $35-45 \%$ [4]. Sepsis and ARDS are closely related, and original author(s) and the source, provide a link to the Creative Commons licence, and indicate if changes were made. The images or other third party material in this article are included in the article's Creative Commons licence, unless indicated otherwise in a credit line to the material. If material is not included in the article's Creative Commons licence and your intended use is not permitted by statutory regulation or exceeds the permitted use, you will need to obtain permission directly from the copyright holder. To view a copy of this licence, visit http://creativecommons.org/licenses/by/4.0/. The Creative Commons Public Domain Dedication waiver (http://creativeco mmons.org/publicdomain/zero/1.0/) applies to the data made available in this article, unless otherwise stated in a credit line to the data. 
most of these patients receive care in intensive care units (ICUs). Up to $50 \%$ of sepsis patients admitted to ICUs develop ARDS [5], and the mortality rate of patients with sepsis and ARDS is greater than that of patients with sepsis alone or ARDS alone [6, 7]. Thus, it is essential to identify the characteristics of sepsis patients who develop ARDS after ICU admission.

Sepsis and ARDS are heterogeneous inflammatory syndromes, and certain phenotypes might be associated with clinical outcomes. Several studies reported that sepsis patients with hyper-inflammation had higher mortality rates [6,7]. Similarly, some studies showed that patients with ARDS and hypo-inflammation might have better clinical outcomes than those with ARDS and hyper-inflammation $[8,9]$. There is also evidence that some pro- and anti-inflammatory biomarkers have prognostic value in patients with sepsis and ARDS [10,11]. Previous studies suggested that biomarkers for ARDS can be classified as epithelial markers (surfactant proteins [SPs], Krebs von den Lungen-6 [KL-6] protein, vascular endothelial growth factor [VEGF], and soluble receptor for advanced glycation end-products [sRAGE]); endothelial markers (angiopoietin-2 [Ang-2]); and inflammatory cytokines (IL-1 $\beta$ and TNF- $\alpha$ ) [12]. However, few studies directly examined the pro- and anti-inflammatory markers of patients with sepsis and ARDS. It is also uncertain whether the levels of pro- and anti-inflammatory cytokines are associated with the development of ARDS in patients with sepsis. Thus, it is necessary to identify novel serum biomarkers that can be used to classify disease severity in patients with sepsis and ARDS.

Fibroblast growth factor 21 (FGF21) is a peptide hormone that regulates energy homeostasis and glucose-lipid metabolism that is synthesized by the liver, adipocytes, pancreas, and brain [13]. The plasma level of FGF21 is greater in patients with metabolic disorders, such as type 2 diabetes, obesity, and mitochondrial diseases, and the level also increases during aging $[14,15]$. Patients with inflammatory reactions, such as sepsis, pancreatitis, and systemic inflammatory response syndrome (SIRS), have increased serum levels of FGF21 [16, 17]. FGF21 apparently reduces oxidative stress by decreasing the level of reactive oxygen species (ROS), repressing nuclear factor kappa B (a pro-inflammatory factor), and reducing apoptosis [18-21]. Siahanidou et al. and our previous study demonstrated that FGF21 has value as a prognostic indicator in patients with sepsis $[22,23]$.

However, the relationship of FGF21 with prognosis in patients with sepsis and ARDS is uncertain. In this prospective cohort study, we aimed to evaluate whether changes in the serum level of FGF21 predict 28-day mortality of ICU patients who have sepsis and ARDS.

\section{Patients and methods Patients}

This prospective cohort study included 231 consecutive patients with sepsis who were admitted to the Department of Critical Care Medicine of Changsha of Traditional Chinese Medicine Hospital (Hunan, China) between January 2019 and December 2020. Sepsis was diagnosed according to the Sepsis-3 criteria [1] and ARDS was diagnosed according to the Berlin definition [2]. All included patients were older than 18 years. The exclusion criteria were cancer, hospitalization or receipt of antibiotics during the preceding 2 weeks, and readmission to the ICU.

All eligible patients were divided into a Sepsis-only group (patients who met the Sepsis-3 criteria and had no evidence of ARDS) and a Sepsis + ARDS group. The Sepsis + ARDS group was further divided into a survival group (patients who survived 28 days after initial diagnosis) and a non-survival group (patients who died within 28 days after diagnosis).

Demographic data (including age and gender) and medical history (including cardiovascular diseases, diabetes mellitus, chronic renal failure, and chronic obstructive pulmonary disease) were recorded. Laboratory values, hemodynamic parameters, ventilator settings, and medications were recorded at disease inception, during the worst period of disease, and during the recovery period. Sequential Organ Failure Assessment (SOFA) scores were calculated. The main clinical outcome measure was 28-day mortality.

Each patient received standard treatment during the ICU stay. This study was approved by the Ethics Committee of Changsha of Traditional Chinese Medicine Hospital and informed consent was obtained from all patients or their guardians.

\section{Cytokine measurements}

Blood samples were collected within $24 \mathrm{~h}$ after diagnosis, during the worst period of disease, and 2-3 days prior to death or discharge (blood samples were collected from the patients every two to three days to monitor for all biochemical markers in our center). The serum levels of FGF21, interleukin 6 (IL-6), IL-10, tumor necrosis factor $\alpha(\mathrm{TNF} \alpha)$, procalcitonin $(\mathrm{PCT})$, and $\mathrm{C}$ reactive protein (CRP) were determined using enzyme-linked immunosorbent assays (ELISAs) from Abcam (USA) according to the manufacturer's instructions. Routine blood tests and blood gas analyses were performed in the hospital's central laboratory. 


\section{Statistical analysis}

The normality of the distribution of a quantitative variable was tested using the Kolmogorov-Smirnov test $(\mathrm{P}>0.10)$. Normally distributed data are expressed as means and standard deviations (SDs), and non-normally distributed data as medians and interquartile ranges (IQRs). Qualitative data are presented as number (\%). For continuous variables, two-group comparisons were performed using Student's $t$-test or the Mann-Whitney $\mathrm{U}$ test, depending on the normality of the distribution. A two tailed $P$ value less than 0.05 was considered statistically significant. For categorical variables, the Chisquare test, Fisher's exact test, or McNemar's test was used as appropriate. The Hotelling $\mathrm{T}^{2}$ test was used to identify the significance of changes in the level of serum FGF21 and other markers.

To identify the risk factors for 28-day mortality of patients in the Sepsis + ARDS group, multiple Cox proportional hazards regression analyses were performed to measure the effects of different variables at three key times: (1) ICU admission; (2) peak of shock; and (3) before death or ICU discharge. The value of each measured parameter $(\mathrm{P})$ is expressed as " $\mathrm{P}_{\mathrm{t}}$, where ' $\mathrm{t}$ ' is the measurement time $(1,2$, or 3$)$; the change in a parameter between two times is expressed as " $\Delta \mathrm{P}_{\mathrm{t} 1-\mathrm{t} 2}$," where ' $\mathrm{t} 1$ ' and ' $\mathrm{t} 2$ ' are the measurement times; and the percentage change in a parameter between two times is expressed as

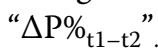

Multivariate receiver operating characteristic curve analysis was performed to compare the different value of FGF21 and SOFA detected at admission as a predictor for 28-day survival. All statistical analyses were performed using SPSS version 24.0 software (IBM SPSS, USA). A two tailed $P$ value below 0.05 was considered significant.

\section{Results}

\section{Comparison of the Sepsis and Sepsis + ARDS groups}

We initially compared the clinical characteristics of patients in the Sepsis-only and the Sepsis + ARDS groups (Additional file 1: Table S1). The Sepsis + ARDS group had a greater respiratory rate, a lower $\mathrm{PaO}_{2} / \mathrm{FiO}_{2}$, a greater SOFA score, and higher levels of lactate (LAC), procalcitonin $(\mathrm{PCT}), \mathrm{C}$-reactive protein $(\mathrm{CRP})$, interleukin 6 (IL-6), tumor necrosis factor $\alpha$ (TNF $\alpha$ ), IL-10, and FGF21 (all $\mathrm{P}<0.05$ ). The serum level of FGF21 was almost two-times greater in the Sepsis + ARDS group.

\section{Comparison of survivors and non-survivors in the Sepsis + ARDS group}

The survivors and non-survivors in the Sepsis + ARDS group had similar age, gender, and underlying diseases (Table 1). At baseline, the non-survivors had a significantly lower mean arterial pressure, hematocrit, and Glasgow Coma Scale (GCS) score; greater heart rate, body temperature, and SOFA score; and greater serum levels of creatinine, LAC, PCT, CRP, IL-6, TNF $\alpha$, IL-10 (Table 1; all $\mathrm{P}<0.05$ ). The serum level of FGF21 was almost four-times greater in the non-survivors (Table 1; all $\mathrm{P}<0.05$ ).

\section{Dynamics of FGF21 in the Sepsis + ARDS group}

We recorded the changes of cytokines and other biomarkers while patients in the Sepsis + ARDS group were in the ICU (Fig. 1). The serum level of FGF21 persistently increased from baseline in the non-survivors, but gradually decreased in the survivors (both $\mathrm{P}<0.05$ ). In addition, pro-inflammatory biomarkers (CRP, PCT, TNF- $\alpha$, IL-6,) and SOFA score also increased in the non-survivors, and decreased in the survivors (all $\mathrm{P}<0.05)$.

Risk factors for 28-day mortality in Sepsis + ARDS patients based on measurements at 3 crucial time points

We initially analyzed variables associated with 28-day mortality based on measurements at 24-48 h after ICU admission (Table 2). Before analysis, main parameters were changed into categorical variables according to their quartile values at baseline (Additional file 2: Table 2). The results indicated that serum FGF21 level, SOFA score, and serum level of CRP were independent risk factors for 28-day mortality (all $\mathrm{P}<0.05$ ).

We then analyzed variables associated with 28-day mortality based on initial measurements and measurements at the peak of shock (Table 3). The results indicated that the $\triangle \mathrm{SOFA}_{2-1}, \mathrm{FGF} 21_{2}$, and $\Delta \mathrm{FGF} 21 \%_{2-1}$ were independent risk factors for 28-day mortality (all $\mathrm{P}<0.05$ ).

We also analyzed variables associated with 28-day mortality based on measurements performed at 2-3 days before death or ICU discharge, measurements at the peak of shock, and initial measurements (Table 4). The results indicated that $\Delta \mathrm{SOFA}_{3-1}, \Delta \mathrm{FGF} 21 \%_{3-1}, \Delta \mathrm{LAC}_{3-1}$, and baseline $\mathrm{CRP}_{1}$ were independent risk factors for 28-day mortality (all $\mathrm{P}<0.05)$.

\section{The predictive value of FGF21 for differentiating} non-survivors and survivors in patients with Sepsis + ARDS We examined the predictive values of FGF21 and SOFA at baseline for differentiating non-survivor and survivor patients with Sepsis + ARDS by use of receiver operating characteristic (ROC) curve analysis (Table 5 and Fig. 2). The results showed that the AUC of FGF21 was 0.856, 
Table 1 Baseline clinical characteristics of patients in the Sepsis + ARDS group who were survivors and non-survivors

\begin{tabular}{|c|c|c|c|}
\hline Parameter & $\begin{array}{l}\text { Non-survivors } \\
(n=42)\end{array}$ & $\begin{array}{l}\text { Survivors } \\
(n=119)\end{array}$ & $P$ value \\
\hline Age (years) & $68 \pm 13$ & $65 \pm 16$ & 0.137 \\
\hline Gender (male/female) & $23 / 19$ & $84 / 35$ & 0.062 \\
\hline Coronary heart disease & $13(31.1 \%)$ & $38(31.9 \%)$ & 0.907 \\
\hline Hypertension & $12(28.6 \%)$ & $36(30.2 \%)$ & 0.838 \\
\hline Type 2 diabetes & $5(11.9 \%)$ & $13(10.9 \%)$ & 0.862 \\
\hline Chronic renal failure & $3(7.1 \%)$ & $8(6.7 \%)$ & 0.926 \\
\hline COPD & $12(28.6 \%)$ & $39(32.8 \%)$ & 0.615 \\
\hline Length of stay & $10(2-22)$ & $9(3-21)$ & 0.578 \\
\hline Mean arterial pressure $(\mathrm{mmHg})$ & $68(35-116)$ & $87(48-120)$ & $<0.001$ \\
\hline Respiratory rate (per min) & $24(12-40)$ & $22(13-38)$ & 0.125 \\
\hline Heart rate (bpm) & $110 \pm 33$ & $98 \pm 20$ & 0.022 \\
\hline Temperature $\left({ }^{\circ} \mathrm{C}\right)$ & $38.1 \pm 1.4$ & $37.2 \pm 0.8$ & 0.019 \\
\hline White blood count $\left(10^{9} / \mathrm{L}\right)$ & $15.0 \pm 6.6$ & $14.3 \pm 7.2$ & 0.682 \\
\hline Platelet count $\left(10^{9} / \mathrm{L}\right)$ & $131(17-364)$ & $178(35-473)$ & 0.055 \\
\hline Hematocrit (\%) & $31.3 \pm 7.0$ & $35.4 \pm 5.7$ & 0.050 \\
\hline $\mathrm{PaO}_{2} / \mathrm{FiO}_{2}(\mathrm{mmHg})$ & $152.1 \pm 55.7$ & $164.6 \pm 54.5$ & 0.021 \\
\hline Total bilirubin ( $\mu \mathrm{mmol} / \mathrm{L})$ & $19.5(4.6-124.7)$ & $19.0(4.8-98.5)$ & 0.183 \\
\hline Creatinine $(\mu \mathrm{mmol} / \mathrm{L})$ & $144.0(44.9-1477.9)$ & $96.4(33.7-507.6)$ & 0.009 \\
\hline Lactate (mmol/L) & $3.7(0.9-18.0)$ & $2.7(1.2-5.6)$ & $<0.0001$ \\
\hline GCS score & $9(3-15)$ & $11(3-15)$ & $<0.0001$ \\
\hline SOFA score & $11(3-21)$ & $6(2-18)$ & $<0.0001$ \\
\hline $\mathrm{CRP}(\mathrm{mg} / \mathrm{L})$ & $134.9(18.6-468.0)$ & $100.7(9.8-200.0)$ & $<0.0001$ \\
\hline $\mathrm{PCT}(\mathrm{ng} / \mathrm{mL})$ & $16.96(1.70-200.00)$ & $3.07(0.15-200.0)$ & $<0.0001$ \\
\hline IL-6 (pg/mL) & $367.7(37.3-498.2)$ & $89.6(2.9-462.6)$ & $<0.0001$ \\
\hline TNF-a (pg/mL) & $63.9(11.1-215.4)$ & $12.3(5.8-102.1)$ & $<0.0001$ \\
\hline IL-10 (pg/mL) & $597.6(68.0-798.7)$ & $108.6(10.7-657.9)$ & $<0.0001$ \\
\hline FGF21 (pg/mL) & $3574.7(327.3-6978.5)$ & $986.6(32.3-6121.3)$ & $<0.0001$ \\
\hline
\end{tabular}

COPD chronic obstructive pulmonary disease

better than that of SOFA (0.832), but this difference was not statistically significant. Besides, the optimal cutoff was $2542 \mathrm{pg} / \mathrm{mL}$ for FGF21 level to better discriminate survivors from non-survivors, with a sensitivity of $69.1 \%$ and specificity of $88.2 \%$. Thus, FGF21 is a promising biomarker for differentiating non-survivors and survivors among patients with Sepsis + ARDS.

\section{Discussion}

In this study, we found that the serum FGF21 level at baseline was greater in ICU patients who had sepsis and ARDS than in those who had sepsis alone. Furthermore, non-survivors in the Sepsis + ARDS group had persistently increasing levels of FGF21 during their ICU stays, but survivors in the Sepsis + ARDS group had persistently declining levels. Notably, our Cox proportional hazards model indicated that an increase in the serum level of FGF21 from the baseline was a significant risk factor for 28-day mortality in the Sepsis + ARDS group.
To our best knowledge, this is the first study to identify the prognostic value of dynamic changes in the serum level of FGF21 in patients with sepsis and ARDS.

Previous studies demonstrated that the serum level of FGF21 was greater in adults and newborns who had sepsis [22, 23]. Our previous study demonstrated that the serum level of FGF21 was positively associated with serum inflammation biomarkers, including PCT, CRP, IL-6, and TNF $\alpha$ [23]. Patients with sepsis and ARDS have a greatly increased inflammatory state. Thus, it is not surprising that the serum level of FGF21 was greater in patients with sepsis and ARDS.

Elevation of inflammatory and anti-inflammatory biomarkers can occur concurrently during the early phase of sepsis [24]. Previous studies reported that FGF21 had anti-inflammatory effects during sepsis [25]. The present study, which compared patients with sepsis alone $v s$. sepsis and ARDS, identified much higher levels of serum FGF21 and pro-inflammatory factors (PCT, CRP, IL-6, 


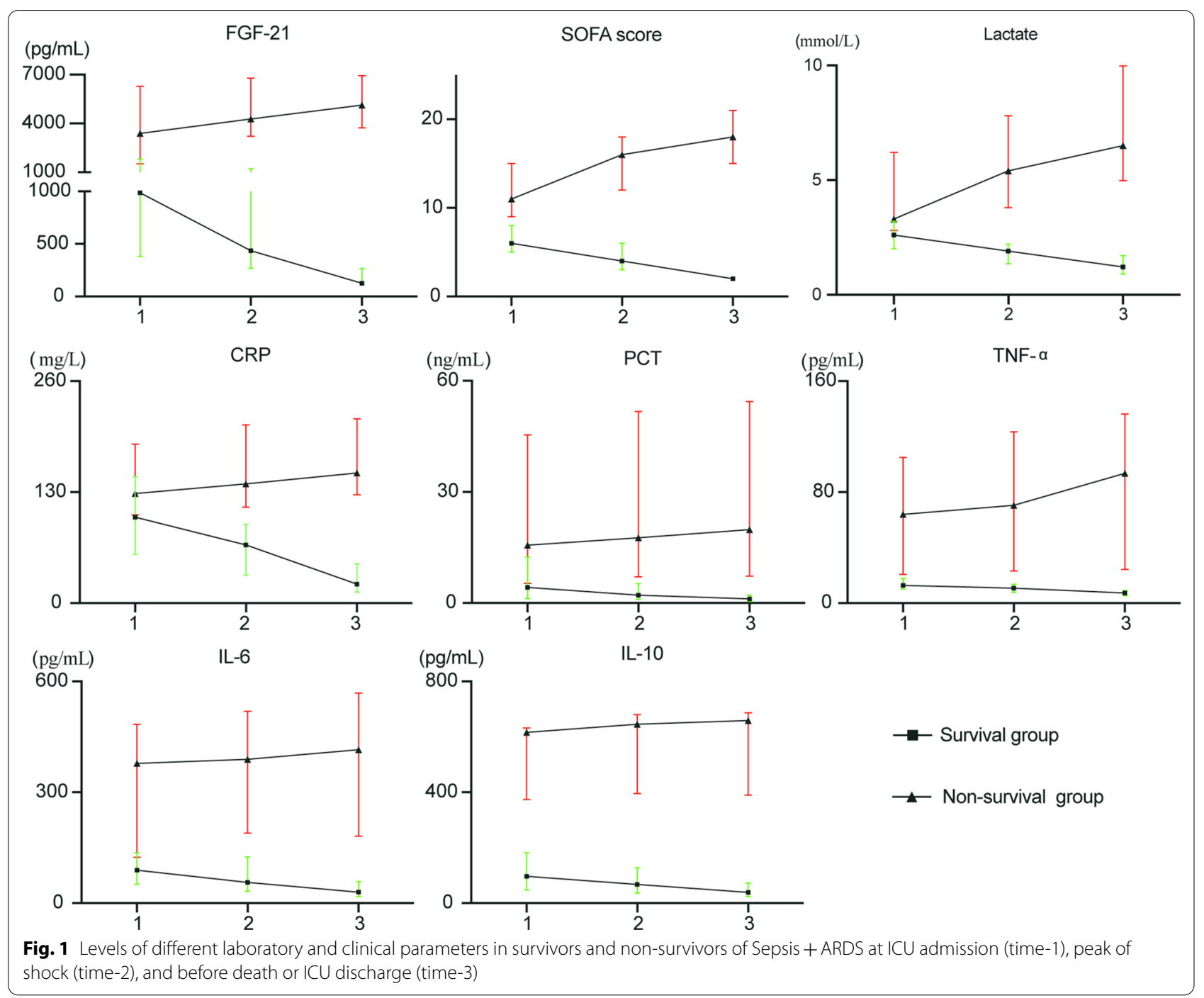

Table 2 Cox proportional hazard analysis of baseline factors associated with mortality

\begin{tabular}{|c|c|c|c|c|}
\hline \multirow[t]{2}{*}{ Variable } & \multicolumn{2}{|l|}{ Univariate Cox model } & \multicolumn{2}{|l|}{ Multivariate Cox model } \\
\hline & $\mathrm{HR}(95 \% \mathrm{Cl})$ & $P$ value & $\mathrm{HR}(95 \% \mathrm{Cl})$ & $P$ value \\
\hline SOFA & $2.973(2.043-4.327)$ & $<0.0001$ & 3.507 (1.770-6.948) & $<0.0001$ \\
\hline FGF21 & $3.388(2.266-5.066)$ & $<0.0001$ & $11.224(3.237-38.917)$ & $<0.0001$ \\
\hline IL-6 & $2.437(1.769-3.358)$ & $<0.0001$ & NA & 0.639 \\
\hline TNF-a & 3.071 (1.926-4.898) & $<0.0001$ & NA & 0.051 \\
\hline IL-10 & $3.220(1.977-5.792)$ & $<0.0001$ & NA & 0.991 \\
\hline PCT & $1.787(1.339-2.385)$ & $<0.0001$ & NA & 0.602 \\
\hline CRP & $1.011(1.008-1.015)$ & $<0.0001$ & $2.029(1.305-3.155)$ & 0.002 \\
\hline LAC & $1.944(1.462-2.584)$ & $<0.0001$ & NA & 0.062 \\
\hline
\end{tabular}

Here and below: NA not applicable 
Table 3 Cox proportional hazard analysis of factors at baseline (1) and the peak of shock (2) that were associated with mortality in the Sepsis + ARDS group

\begin{tabular}{|c|c|c|}
\hline \multirow[t]{2}{*}{ Variable } & \multicolumn{2}{|l|}{ Multiple Cox model } \\
\hline & HR $(95 \% \mathrm{Cl})$ & $P$ value \\
\hline SOFA $_{1}$ & $1.247(1.096-1.418)$ & 0.001 \\
\hline SOFA2 & NA & 0.956 \\
\hline$\triangle \mathrm{SOFA}_{2-1}$ & NA & 0.692 \\
\hline$\triangle \mathrm{SOFA}_{2-1}$ & NA & 0.617 \\
\hline FGF21 1 & NA & 0.053 \\
\hline FGF2 $1_{2}$ & $1.001(1.000-1.001)$ & $<0.0001$ \\
\hline$\Delta$ FGF21 $\%_{2-1}$ & $15.269(1.622-143.712)$ & 0.017 \\
\hline $\mathrm{LAC}_{1}$ & NA & 0.730 \\
\hline $\mathrm{LAC}_{2}$ & NA & 0.652 \\
\hline$\triangle \mathrm{LAC} \%_{2-1}$ & NA & 0.812 \\
\hline
\end{tabular}

Here and below: the difference in the value of a parameter $(P)$ at different times is expressed as " $\Delta \mathrm{P}_{\mathrm{t} 1-\mathrm{t} 2}$ ", where ' $\mathrm{t} 1$ ' and ' $\mathrm{t} 2$ ' are the measurement times; and the percentage change in a parameter at different times is expressed as $\Delta \mathrm{P} \%_{\mathrm{t} 1-\mathrm{t} 2}$

Table 4 Cox proportional hazard analysis of factors at baseline (1), peak of shock (2), and at death or ICU discharge that were associated with mortality in the Sepsis + ARDS group

\begin{tabular}{|c|c|c|}
\hline \multirow[t]{2}{*}{ Variable } & \multicolumn{2}{|l|}{ Multiple Cox model } \\
\hline & $\mathrm{HR}(95 \% \mathrm{Cl})$ & $P$ value \\
\hline $\mathrm{SOFA}_{1}$ & NA & 0.664 \\
\hline $\mathrm{SOFA}_{2}$ & NA & 0.735 \\
\hline SOFA $_{3}$ & $1.148(1.023-1.289)$ & 0.019 \\
\hline$\triangle \mathrm{SOFA}_{2-1}$ & NA & 0.399 \\
\hline$\triangle \mathrm{SOFA}_{3-2}$ & NA & 0.955 \\
\hline$\triangle \mathrm{SOFA}_{3-1}$ & NA & 0.625 \\
\hline FGF21 & NA & 0.294 \\
\hline FGF2 $1_{2}$ & NA & 0.197 \\
\hline $\mathrm{FGF} 21_{3}$ & NA & 0.384 \\
\hline$\Delta \mathrm{FGF} 21 \%_{2-1}$ & NA & 0.272 \\
\hline$\Delta \mathrm{FGF} 21 \%_{3-2}$ & NA & 0.413 \\
\hline$\Delta \mathrm{FGF} 21 \%_{3-1}$ & $25.760(1.482-447.610)$ & 0.026 \\
\hline $\mathrm{LAC}_{1}$ & NA & 0.838 \\
\hline $\mathrm{LAC}_{2}$ & NA & 0.834 \\
\hline $\mathrm{LAC}_{3}$ & NA & 0.830 \\
\hline$\triangle \mathrm{LAC} \%_{2-1}$ & NA & 0.435 \\
\hline$\Delta \mathrm{LAC} \%_{3-2}$ & 0.693 & 0.693 \\
\hline$\Delta \mathrm{LAC}_{3-1}$ & $1.512(1.041-2.196)$ & 0.030 \\
\hline $\mathrm{CRP}_{1}$ & $1.008(1.003-1.014)$ & 0.003 \\
\hline
\end{tabular}

and TNF $\alpha$ ) in the Sepsis + ARDS group. This suggests a more intense interaction between pro-inflammatory and anti-inflammatory factors in patients who have sepsis and ARDS.
Immune cells function in the early stage of ARDS, so the focused biomarkers for ARDS in this stage are inflammatory cytokines, such as IL- $1 \beta$ and TNF- $\alpha$ which are mainly related to exudation and migration of inflammatory. However, the anti-inflammatory cytokines also play a critical role in this stage and should not be ignored. Besides it is also critical to identify novel biomarkers for late stage of ARDS which is not well investigated currently. FGF21 has anti-inflammatory and anti-oxidative stress effects [18-21]. This motivated our interest in the relationship between the dynamics changes of FGF21 and the prognosis of ARDS during sepsis in the present research.

In this study, we found that FGF21 is a promising prognostic biomarker for differentiating non-survivors and survivors among patients with Sepsis + ARDS (Table 1). Our analysis of disease progression in patients with sepsis and ARDS indicated the non-survivors had continuously increased serum levels of FGF21, and this was accompanied by persistent increases in the levels of multiple proinflammatory cytokines. In contrast, the survivors had gradually decreased levels of FGF21 and of pro-inflammatory cytokines. The presence of higher levels of proand anti-inflammatory biomarkers in the non-survivors is consistent with the presence of increased physiological stress.

Because the dynamic changes of pro- and anti-inflammatory markers appeared to be of vital importance in the progression of patients with sepsis and ARDS, we were interested in the following question: Which parameters can be used to predict the mortality of patients with sepsis and ARDS? Thus, we used Cox regression analysis to assess the prognostic value of biomarkers that were measured at admission to the ICU, at the peak of shock, and before death (non-survivors) or before ICU discharge (survivors). Our measurements at admission indicated that increased FGF21, LAC, and SOFA score were associated with poor prognosis in patients with sepsis and ARDS. This result is consistent with results from our previous study [23].

Our measurements at the peak time of shock (typically 1 week after ICU admission) indicated that the level of FGF21 at the peak did not show significant difference, but the percentage increase in the FGF21 from baseline $\left(\Delta\right.$ FGF21 $\left.\%_{2-1}\right)$ value had a much greater HR $(15.269$ [95\% CI 1.622-143.712] vs. 1.247 [95\% CI 1.096-1.418]). In other words, based on measurements at baseline and the peak of shock, we identified the percentage increase of FGF21 as the best predictor of poor prognosis in patients with sepsis and ARDS. The FGF21 at the peak may serve as a good predictor with enlarged sample size. The large changes in the serum level of FGF21 from admission to the peak of shock reflect the dynamics of pro- and 
Table 5 Accuracy of FGF21 and SOFA in differentiating non-survivors and survivors among patients with Sepsis + ARDS

\begin{tabular}{lllllll}
\hline Parameter & $\begin{array}{l}\text { Areas under curve } \\
\text { (AUC) }\end{array}$ & 95\%Cl & Sensitivity (\%) & Specificity (\%) & $\begin{array}{l}\text { Positive predictive } \\
\text { value (\%) }\end{array}$ & $\begin{array}{l}\text { Negative } \\
\text { predictive } \\
\text { value (\%) }\end{array}$ \\
\hline FGF21 & $0.856^{*}$ & $0.792-0.906$ & 69.1 & 88.2 & 68.2 & 87.5 \\
SOFA & 0.832 & $0.766-0.887$ & 71.43 & 80.7 & 61.3 & 86.8 \\
\hline
\end{tabular}

* Comparison of AUC between FGF21 and SOFA, P value $=0.5465$

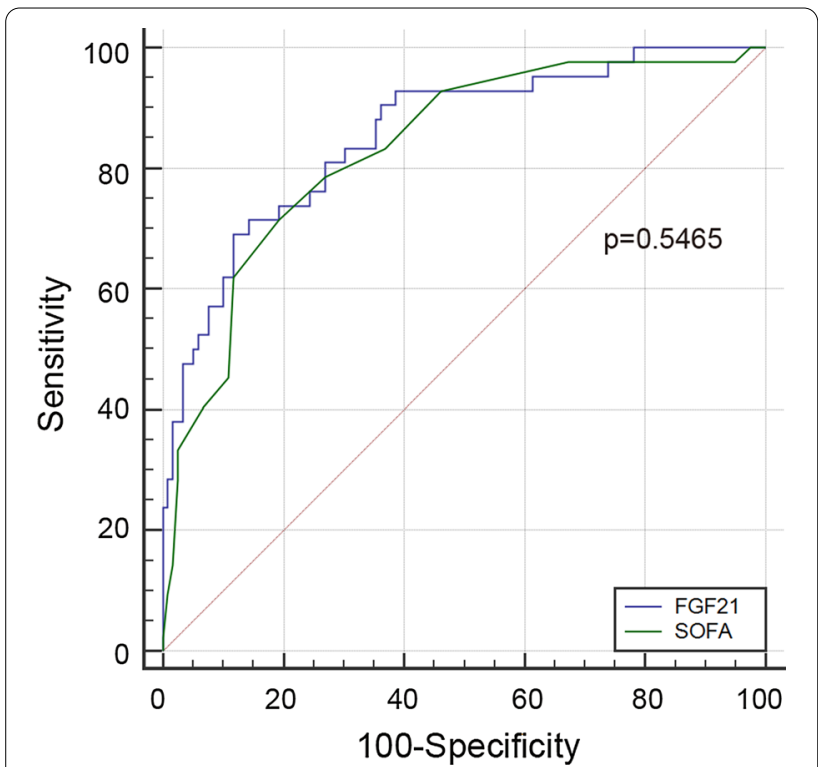

Fig. 2 Receiver operating characteristic curve (ROC) of FGF21 vs. SOFA in predicting 28-day mortality of critical patients with sepsis + ARDS

anti-inflammatory factors in patients with sepsis and ARDS.

We also analyzed the relationship of the final clinical measurements (before death or ICU discharge) with patient prognosis. We found that the $\Delta \mathrm{FGF} 21 \%_{3-1}$, $\triangle \mathrm{LAC}_{3-1}$, and $\triangle \mathrm{SOFA}_{3-1}$, and $\mathrm{CRP}_{1}$ were significant prognostic factors. Notably, $\triangle \mathrm{FGF} 21 \%_{3-1}$ was best predictor of poor prognosis $(\mathrm{HR}=25.760$ [95\% CI $1.482-$ 447.610]). This confirms that a large increase in the serum level of FGF21, either between the first and second measurements or between the first and third measurements, was strongly associated with 28-day mortality in patients with sepsis and ARDS. At the physiological level, this may be attributed to the anti-inflammation, anti-apoptotic, and anti-oxidative effect of FGF21 during sepsis.

\section{Limitations}

First, this was a single-center study, and the total number of patients with sepsis and ARDS was relatively small. However, we have been pursuing this topic and collecting the records of sepsis patients for several years. A large and multi-center study should be performed to verify our observations. Second, because pro-inflammatory and anti-inflammatory factors both increased during sepsis, it is possible that other unmeasured anti-inflammatory cytokines might have also played a crucial role. We are currently analyzing the prognostic value of other anti-inflammatory biomarkers in sepsis patients, and plan further comparisons of their functions in patients with sepsis and ARDS. Third, some sepsis patients experience a hypoinflammation phenotype. Most of our patients experienced hyper-inflammation, and this may be attributable to our exclusion criteria, such as exclusion of patients with cancer. The effect of changes in the serum level of FGF21 on the prognosis of patients with sepsis and ARDS who have hypo-inflammation is a topic that needs further study.

\section{Conclusion}

An increase in the serum level of FGF21 after ICU admission of patients who have sepsis and ARDS is associated with a significantly increased risk of 28-day mortality.

\section{Abbreviations}

ARDS: Acute respiratory distress syndrome; ICUs: Intensive care units; FGF21: Fibroblast growth factor 21; SOFA: Sequential Organ Failure Assessment; IL-6: Interleukin 6; IL-10: Interleukin 10; TNFa: Tumor necrosis factor a; PCT: Procalcitonin; CRP: C reactive protein; LAC: Lactate; GCS: Glasgow Coma Scale; ROC: Receiver operating characteristic curve.

\section{Supplementary Information}

The online version contains supplementary material available at https://doi. org/10.1186/s12931-021-01778-w.

Additional file1: Table S1. Baseline clinical characteristics of patients in the Sepsis + ARDS group and the Sepsis-only group.

Additional file2: Table S2. Quartiles of $\triangle$ SOFA, Lac, FGF21, PCT, TNF-a, $\mathrm{IL}-6, \mathrm{IL}-10$, and CRP used in the Cox regression analysis.

Acknowledgements

Not applicable. 


\section{Authors' contributions}

$Y L, T L, J F H, C K L, Z L D$ and $Y C$ finished clinical studies and collected primary data. THZ and XYC analyzed the data. XL finished the experiment, and performed statistic analysis, and wrote the manuscript. HS participated in the design of the study and edited the manuscript. YT and ZXZ conceived of the study, and participated in its design and coordination. All authors read and approved the final manuscript.

\section{Funding}

This study was sponsored by the Natural Science Foundation of Hunan Province, China (No.2019JJ80051 and No.2019JJ80061).

\section{Availability of data and materials}

Data will not be shared with a reason.

\section{Declarations}

Ethics approval and consent to participate

The study was approved by the Ethics Committee of Changsha of Traditional Chinese Medicine Hospital.

\section{Consent for publication}

An informed consent form was obtained from all the patients or their guardians.

\section{Competing interests}

The authors declare that they have no competing interests.

Received: 22 March 2021 Accepted: 10 June 2021

Published online: 22 June 2021

\section{References}

1. Singer M, Deutschman CS, Seymour CW, Shankar-Hari M, Annane D, Bauer $M$, et al. The third international consensus definitions for sepsis and septic shock (sepsis-3). JAMA. 2016;315:801-10.

2. Ranieri VM, Rubenfeld GD, Thompson BT, et al. Acute respiratory distress syndrome: the Berlin Definition. JAMA. 2012. https://doi.org/10.1001/ jama.2012.5669.

3. Lever A, Mackenzie I. Sepsis: definition, epidemiology, and diagnosis. BMJ. 2007:335(7625):879-83.

4. Bellani G, Laffey JG, Pham T, et al. Epidemiology, patterns of care and mortality for patients with acute respiratory distress syndrome in intensive care units in 50 countries. JAMA. 2016;315(8):788.

5. Hudson LD. Clinical risks for development of the acute respiratory distress syndrome. Am J Respir Crit Care Med. 1995;151:293-301.

6. Hatfield KM, Dantes RB, Baggs J, et al. Assessing variability in hospitallevel mortality among U.S. Medicare Beneficiaries with hospitalizations for severe sepsis and septic shock*. Crit Care Med. 2018;46(11):1753-60.

7. Sevransky JE, Martin GS, Carl S, et al. Mortality in sepsis versus non-sepsis induced acute lung injury. Crit Care. 2009;13(5):R150.

8. Ricarte-Bratti JP, Brizuela NY, et al. IL-6, MMP 3 and prognosis in previously healthy sepsis patients. Revista de la Facultad de Ciencias Medicas (Cordoba, Argentina). 2017;74(2):99.
9. Anderson BJ, Calfee CS, Liu KD, et al. Plasma sTNFR1 and IL8 for prognostic enrichment in sepsis trials: a prospective cohort study. Crit Care. 2019. https://doi.org/10.1186/s13054-019-2684-2.

10. Calfee CS, Delucchi K, Parsons PE, et al. Latent class analysis of ARDS sub phenotypes: analysis of data from two randomized controlled trials. Lancet Respir Med. 2014;2(8):611.

11. Bos LD, Schouten LR, Van Vught LA, et al. Identification and validation of distinct biological phenotypes in patients with acute respiratory distress syndrome by cluster analysis. Thorax. 2017:72(10):876-83.

12. Spadaro S, Park M, Turrini $C$, et al. Biomarkers for Acute Respiratory Distress syndrome and prospects for personalized medicine. J Inflamm. 2019. https://doi.org/10.1186/s12950-018-0202-y.

13. Xie T, Leung PS. Fibroblast growth factor 21: a regulator of metabolic disease and health span. Am J Physiol Endocrinol Metab. 2017;313(3):E292-302.

14. Staiger H, Keepers M, Berti L, et al. Fibroblast growth factor 21 - metabolic role in mice and men. Endocr Rev. 2017. https://doi.org/10.1210/er. 2017-00016.

15. Tezze C, Romanello V, Desbats MA, et al. Age-associated loss of OPA1 in muscle impacts muscle mass, metabolic homeostasis, systemic inflammation, and epithelial senescence. Cell Metab. 2017. https://doi.org/10. 1016/j.cmet.2017.04.021.

16. Gariani K, Drifte G, Dunn-Siegrist I, Pugin J, Jornayvaz FR. Increased FGF21 plasma levels in humans with sepsis and sirs. Endocr Connect. 2013;2(3):146-53.

17. Shenoy VK, Beaver KM, Fisher FM, et al. Elevated serum fibroblast growth factor 21 in humans with acute pancreatitis. PLoS ONE. 2016;11(11):e0164351.

18. Planavila A, Redondo-Angulo I, Ribas F, Garrabou G, Casademont J, Giralt $M$, et al. Fibroblast growth factor 21 protects the heart from oxidative stress. Cardiovasc Res. 2015;106:19-31.

19. Planavila A, Redondo I, Hondares E, et al. Fibroblast growth factor 21 protects against cardiac hypertrophy in mice. Nat Commun. 2013;4(3):2019.

20. Hondares E, Iglesias R, Giralt A, Gonzalez FJ, Giralt M, Mampel T, et al. Thermogenic activation induces FGF21 expression and release in brown adipose tissue. J Biol Chem. 2011;286:12983-90.

21. Jeanson Y, Ribas F, Galinier A, Arnaud E, Ducos M, Andre M, et al. Lactate induces FGF21 expression in adipocytes through a p38-MAPK pathway. Biochem J. 2016;473:685-92.

22. Siahanidou T, Margeli A, Bourika V, et al. Association of fibroblast growth factor 21 plasma levels with neonatal sepsis: preliminary results. Clin Chem Lab Med. 2019:57(5):e83-5.

23. Xing $L$, Zexiang $Z$, Tinghong $Z$, et al. Early increases in serum FGF21 levels predict mortality of septic patients. Cytokine. 2018;111:428-33.

24. Tamayo E, Fernandez A, Almansa R, Carrasco E, Heredia M, Lajo C, et al. Pro- and anti-inflammatory responses are regulated simultaneously from the first moments of septic shock. Eur Cytokine Netw. 2011:22:82-7.

25. Feingold KR, Grunfeld C, Heuer JG, Gupta A, Cramer M, Zhang T, et al. FGF21 is increased by inflammatory stimuli and protects leptindeficient ob/ob mice from the toxicity of sepsis. Endocrinology. 2012;153:2689-700

\section{Publisher's Note}

Springer Nature remains neutral with regard to jurisdictional claims in published maps and institutional affiliations. 